\title{
HIGH SPEED SUBMARINE OPTICAL FIBER COMMUNICATION SYSTEM: PRESSURE AND TEMPERATURE EFFECTS
}

\author{
A. A. MOHAMMED \\ Faculty of Electric Engineering, Menouf, 32951, EGYPT
}

\begin{abstract}
In the present paper, the performance of high speed submarine optical fiber cable systems is investigated, taking into account both the pressure and the temperature effects. Both the pressure and the temperature are depth-dependent variables, while both the spectral losses and the dispersion effects are temperature as well as wavelength dependent variables. Two important cases with real fibers are processed: a case with dispersion cancellation and a case without dispersion cancellation. It is found that the ocean pressure (due to the depth) shifts the dispersion-free wavelength towards the third communication window. In general, as the depth increases the maximum transmitted bit rate increases in the range of interest. The system capacity as well as the spectral losses, and the dispersion effects are parametrically investigated over wide-range ranges of the set of affecting parameters \{wavelength, ocean depth (and consequently the ocean pressure and temperature), and the chemical structure\}.
\end{abstract}

Key Words: Submarine Optical Fiber, Undersea Optical Communication, Pressure and Temperature Effects, Transoceanic Optical Communications

\section{INTRODUCTION}

Submarine optical fiber cables have been employed in a number of countries to realize long-length high-bitrate optical fiber transmission systems utilizing the second and the third optical windows for one decade. The first generation of undersea systems was installed in 1988 and provided service between North America and Europe across the Atlantic Ocean and between North America and Japan across the Pacific Ocean. The second generation of twice capacity was installed in 1991 and spanned the North Atlantic from U.S. and Canada to U.K. , France, and Spain with multiple landing that was accomplished by Undersea Branching Multiplexers (UBM) capable of exchanging traffic between fiber pairs ${ }^{[1,2]}$. The first sea trial of submarine optical fiber cable employing dispersion shifted fibers was described in Ref. [2], where it included fiber, a structural design for dispersion shifted fiber, cable manufacturing results, fiber splicing, cable laying results, and a high accuracy measurement technique for optical losses and chromatic dispersion. Based on these, the feasibility of long-span and high capacity $1.5-\mu \mathrm{m}$ submarine optical fiber cable was demonstrated. Two types of fibers can be considered feasible: conventional fiber with $1.3 \mu \mathrm{m}$ zero dispersion wavelength or dispersion shifted fiber with zero dispersion in the $1.5 \mu \mathrm{m}$ wavelength region. Based on transmission experiments using Fabry-Perot laser diode (FP-LD) and distributed feedback laser diode (DFB-LD) optical source, a next generation of optical submarine cable communication systems are designed to operate at $1.55 \mu \mathrm{m}{ }^{[3]}$. Different combinations of fibers and optical sources are investigated to achieve a repeater spacing more than $100 \mathrm{~km}$ at $280 \mathrm{Mbits} / \mathrm{sec}$. Recently, there have been some remarkable reports on coherent optical communication systems which make use of phase, frequency and polarization characteristics of lightwave. Polarization fluctuation characteristics of optical fiber submarine cables under $8-\mathrm{km}$ deep sea environmental conditions, optical fiber submarine cable coupling under periodic variable tension, and cable performance during and after installation were analyzed $^{[4]}$.

The history of undersea cable technology has been marked by a few, long periods during which the undersea cable network grew slowly and the technology improved gradually and cautiously. The quiet periods were separated by breakthrough periods during which the technology changed radically, with the previous technology essentially being completely abandoned. The first period-about one hundred years long-was that of single-channel telegraph cables. An extensive cable network was installed during this period, many of which still lie on the sea bottom today (and which have to be removed before new cables can be deployed on the same route). The second period, from about 1950 to 1985 , was characterized by the use of coaxial cables carrying analog telephone traffic. The introduction of electronic vacuum tubes, and later on, of semiconductors in the repeaters permitted the transmission of multiplexed $4-\mathrm{kHz}$ channels, whose number grew from 36 to 4000 per cable, using high frequency carrier techniques. Increasing the capacity mode, it is necessary to increase the diameter of the coaxial cable and to reduce repeater spacing, thus increasing the systems cost. Like in the previous period, the cables were essentially used in point-topoint systems and a new transatlantic cable was installed approximately every four years.

It was during this period that satellites appeared which competed with undersea cable systems. The first satellite used for intercontinental transmission was launched by INTELSAT(I) in 1965 with a rather low capacity of 240 channels. However, satellite technology made significant progress as well, and the 
capacity of INTELSAT (V), launched in 1981, reached 10000 circuits. Numerous economic comparisons were made between satellite and undersea cable systems, with ambiguous and often contradictory results. In fact, a complementary relationship developed between satellite and undersea cable systems because of the advantage of mutual restoration in case of failures of either system. The two systems, however, offer different opportunities: undersea cables are well suited for high-capacity, point-to-point transmission whereas satellites provide more flexibility ${ }^{[5]}$. The advent of optical-fiber cable technology and of optical amplifiers has provided a strong incentive for the development of new undersea cable networks. As a result, significant difficulties have been surmounted, including the problem of the accumulation over very long distances of the effect of imperceptible degradation phenomena.

The above problems are currently being solved for 5 Gbits/sec undersea cable systems, and solutions are being sought for improving the systems performance and economy. Further improvements are still foreseeable, but it is not yet clear when they will be introduced. For instance, ultra-low-loss halide fibers operating at longer wavelengths may permit the achievement of transoceanic distances without any repeaters, the only loss being that of splices. For such fibers, the main foreseeable impairments would come from chromatic-and polarization-mode dispersion ${ }^{[5]}$.

The main trends for the evolution of global undersea cable networks are seen as follows:

1) Since several fiber pairs may be present in the same cable, but can be separated inside branching units, network planners no longer need to build point-to point links, but may build more complex undersea networks, so as to connect through one single transoceanic cable several landing stations on each side of an ocean, or to connect numerous stations to an optical undersea backbone parallel to the coast of a continent.

2) The possibility of using undersea branching multiplexers inside the branching unit (using electronic time division multiplexing (TDM) like in the TAT 9 technology, and probably all-optical wavelength division multiplexing (WDM) in the future, permits further development of these undersea networks, without increasing the number of fibers per cable.

3) The ever-increasing capacity of optical undersea cables is no longer commensurate with that of satellite transmission systems; therefore, in case of a cable fault, traffic can no longer be restored through satellites. The undersea cable network must, therefore, include its own restoration facilities using, for example, ring architectures. Moreover, a new "regional" undersea network, involving TAT 12 and TAT 13, may include its own network protection system which operates nearly instantaneously.

4) Optical undersea cable systems are now being deployed in many parts of the world, and access to undersea cables is becoming available from, virtually, any location. Consequently, undersea cables are no longer considered point-to point links, but as elements of a giant global network. Undersea cable operators aim at exploiting this situation by optimizing, on a real-time basis, the flow of traffic over these networks, taking into account local time differences and changes in the availability of cables.

At present, new undersea cable network development not only relies on technological progress in optical transmission but also on the development of an undersea Telecommunication Network Management System (NMS) which should benefit from recent standards work aimed at harmonizing the NMS for terrestrial transmission systems based on the Synchronous Digital Hierarchy.

The advent of the optical amplifiers has removed the loss limitation of the fiber in the conventional undersea systems using 3R (retiming, reshaping, and regenerating) repeaters, and it has introduced new design criteria for the undersea lightwave systems. The accumulation of the small impairment factors that was negligible in the conventional system becomes significant to determine the transmission performances of the amplified system. The fiber nonlinearity is a distinctive limitation factor that dominates the transmission performance of the amplified system, although it was not a limitation factor in the conventional system.

The recent progress of the undersea lightwave cable systems employing optical amplifier repeaters was described in [6]. The limitation factors and polarization-dependent characteristics of the amplified system are described. The system demonstrations with conventional IM-DD technology are presented using both recirculating loop and straight fiber transmission line. The system maintenance method is also explained briefly. Future technologies adopting the WDM or the optical solitons are also discussed in [6].

The erbium-doped fiber amplifier (EDFA), a research curiosity in the late 1980's, was the backbone of undersea lightwave communications by the mid1990's. Erbium-doped fiber amplifiers have a profound impact on the design, operation, and performance of transoceanic cable transmission systems. The first amplifier systems which was installed in the mid1990's increased the capacity of undersea systems four-to eight fold over the most advanced digital fiber system in use at that time. Later generations of erbiumdoped fiber amplifier systems promise to satisfy the international transmission needs into the $21^{\text {st }}$ century [7].

A new modulation format for optical time domain reflectometry (OTDR) to eliminate optical surge and improve OTDR performance in optical amplifier submarine transmission systems was proposed where the modulation format, FSK-ASK, uses ashore highpower probe pulse and a long dummy pulse, with the slow gain dynamics of erbium-doped fiber amplifiers. Both pulses experience an identical gain, equal to the loss of a span, so that the probe pulse maintains its high 
power and does not develop into an optical surge. Fault location experiments verify a theoretical prediction that FSK-ASK improves the signal-to-noise ratio (SNR) of OTDR by an amount as large as the power ratio of the probe to the dummy signal. They also confirm the elimination of the optical surge caused by conventional OTDR using a solitary probe pulse. An FSK-ASK OTDR is applied in a commercial submarine amplifier transmission system which has a total transmission length of $890 \mathrm{~km}$ and a repeater spacing of $90 \mathrm{~km}$. These field trial results show that subtle fiber anomalies can be located, with a spatial resolution of 1 $\mathrm{km}$, along the entire length of the amplifier transmission system from a terminal end ${ }^{[8]}$.

The FSA submarine optical amplifier system developed for commercial use was designed to transmit 2.5 and $10 \mathrm{Gbits} / \mathrm{sec}$ signals flexibly. Its repeater spacing was $90 \mathrm{~km}$. It contains six line pairs to yield a maximum transmission capacity of $60 \mathrm{Gbits} / \mathrm{sec}$. Its system configuration, and the characteristics of its fibers and optical amplifiers which realize effective dispersion and optical passband management, are introduced. Its performance was discussed with regard to the parameters significant in optically amplified transmission: evolution of zero dispersion wavelength (ZDW), polarization mode dispersion (PMD), and fiber-nonlinearity induced impairments. Signal-tonoise ratios for 2.5 and $10 \mathrm{Gbits} / \mathrm{sec}$ signal transmission were measured and the improvements offered by polarization scrambling were also discussed. Finally, ZDW, PMD, and SNR characteristics of the system after installation were reported ${ }^{[9]}$.

Optical cables have been installed in BT telecommunications networks for about 10 years. The continued satisfactory operation of these optical networks is dependent on the ability of the cable to withstand the environmental conditions found in service. The most common environmental degradation mechanisms such as fiber bending loss, hydrogen degradation loss and stress corrosion have been described in [10]. The effects of these conditions on cable design have been discussed. Designers at present base optical cable structures on theoretical analysis and laboratory results. This leads to a conservative design philosophy but the situation will improve as more field experience is gained.

Today's transoceanic systems can span up to 10,000 $\mathrm{km}$, encountering the full range of ocean environments: $6 \mathrm{~km} \mathrm{ft}$ depths, undersea mountain ranges and eventual landing on different shoreline profiles. Traffic at data rates of $>2.5 \mathrm{Gbit} / \mathrm{sec}$ through thousands of kilometers of submarine cable and hundreds of undersea repeaters, were processed.

The tremendous need for system reliability, combined with rugged, remote environments in which they are deployed, places a premium on quality. At STC Submarine Systems, a subsidiary of Northern Telecom, conformance with ISO 9000 standards has played a major role in this quality effort, so necessary to maintaining a presence in submarine telecommunications business ${ }^{[10]}$.

Compared to electronic regeneration, direct optical amplification using EDFAs, possesses many advantages for long-haul repeater and can be quite simply configured regrardless of the line signal bit rate. This feature becomes more significant as the line signal bit rate increases over several Gbits/sec at which speed electronic regeneration requires high-speed electronic circuits, thereby resulting in an increase in hardware cost and power consumption. Therefore, optical amplifiers are particularly useful in submarine repeaters that have severe space and power constraints. Second, optical amplifiers are flexible regarding bitrate and modulation format, and support wavelength division multiplexed signal transmission. These features suggest that installed systems can be upgraded by changing only the terminal equipment. This advantage is inestimable in submarine transmission systems whose undersea components are virtually impossible to alter once they are laid ${ }^{[11]}$.

A knowledge of the refractive index as a function of pressure is necessary in evaluation of chromatic dispersions at different pressures (depths). Since it plays a vital role in the OFCS in particular for the undersea submarine optical fiber cables which are used to transmit optical signals from one continent to other.

The pressure-dependent Sellemeier coefficients are essential to characterize the optical design parameters for the optical fiber communication systems under deep sea environmental conditions. These coefficients are calculated for densified silica glass for the first time to compute the pressure dependence of material dispersion at any wavelength from the ultraviolet to $1.71 \mu \mathrm{m}$. The zero dispersion wavelength $\lambda_{\mathrm{o}}(1.2725$ $\mu \mathrm{m}$ at $0.1 \times 10^{6} \mathrm{Nm}^{-2}$ ) varies linearly with pressure, $\mathrm{P}$, where the rate of variation of $\lambda_{0}$ with the pressure $\left(\mathrm{d} \lambda_{\mathrm{o}} / \mathrm{dp}\right)$ is $0.0027 \mathrm{~nm} /\left(10^{6} \mathrm{Nm}^{-2}\right)$. The calculated value is approximately one-third of the experimental value of $0.0076 \mathrm{~nm} /\left(10^{6} \mathrm{Nm}^{-2}\right)$ for a germanium-doped dispersion shifted fiber having $\lambda_{\mathrm{o}}=1.5484 \mu \mathrm{m}$ and $0.0070 \mathrm{~nm} /\left(10^{6} \mathrm{Nm}^{-2}\right)$ for a pure silica-core fiber cable having $\lambda_{0}=1.2860 \mu \mathrm{m}$. Since the refractive indices are increased with pressure, the negative value of shift of the zero-dispersion wavelength is erroneous. The explanations are due to Ge-doping in silica glass, a possible temperature fluctuation of $0.16{ }^{\circ} \mathrm{C}$ in the pressure-dependent measurement system of the zero dispersion wavelength and different experimental conditions of the silica glass and the optical fibers. This anomaly can also be attributed to the internal strain development at the core-cladding and fiber-jacketing boundaries due to pressure, which shows a larger experimental value. It accounts for the experimental values satisfactorily ${ }^{[12]}$.

EDFAs have several characteristics that make them an ideal choice for many lightwave applications, including long-haul transmission. EDFAs can be made with a variety of gains in the low-loss $1550 \mathrm{~nm}$ wavelength window of telecommunications fibers, with nearly ideal noise performance, EDFAs amplify highspeed signals without distortion or crosstalk between wavelengths, even when the amplifier is operated deep in gain compression. EDFAs are a fiber device, splicing naturally with telecommunication fiber, and are insensitive to polarization effects, and the EDFAs 
pump laser diodes that are manufactured from the same InGaAsP semiconductor family that has proven undersea reliability with millions of device hours on $1.3 \mu \mathrm{m}$ and $1.55 \mu \mathrm{m}$ signal lasers. All other EDFA components are passive devices, making amplifier designs simple and ensuring that EDFAs can be made with high reliability.

Given these advantages, the next generation of undersea cable transmission systems will use EDFAs in repeaters to boost signals periodically as they travel across the world's oceans. EDFAs will make possible a significant improvement in the capacity of undersea lightwave cable systems installed in the mid 1990's. The first EDFA-based systems will use the NonReturn-to-Zero (NRZ) modulation format at bit rates up to $5 \mathrm{Gbit} / \mathrm{sec}$ with dispersion-shifted fiber that is matched to the operating wavelength. AT\&T, with its partners, installed and operated transoceanic cable systems using EDFA repeaters (1995-1996) in the Atlantic and Pacific [13].

In the present paper, the high speed transmission fiber cable are investigated where both the thermal and pressure effects due to the depth are taken into account. Section II deals with the basic model and analysis where depth-dependent correlations of both the temperature and the pressure are cast, while section III handles the results and a discussion based on the basic model. Finally, Section IV summarizes the major conclusion.

\section{BASIC MODEL AND ANALYSIS:}

Based on reported data of [12], the pressure-dependent Sellemeier coefficients and material dispersions for silica fiber glass, will be cast under the form:

$$
n^{2}(\lambda, T, P)=n^{2}(\lambda, T) f(P, \lambda)
$$

where $\mathrm{n}$ is the refractive index, $\lambda$ is the optical wavelength, $\mathrm{T}$ is the ambient temperature, $\mathrm{P}$ is the pressure. Ghosh et al. [12] cast the following:

$$
n^{2}(\lambda, P)=A+\frac{B \lambda^{2}}{\lambda^{2}-C}+\frac{D \lambda^{2}}{\lambda^{2}-E}
$$

with:

$$
\begin{aligned}
& A=1.29552+9.86385 \times 10^{-6} P+0.544763 \times 10^{-8} P^{2}(3) \\
& B=0.809872+42.0899 \times 10^{-6} P-1.71823 \times 10^{-8} P^{2} \\
& C=1.07945 \times 10^{-2}-0.56693 \times 10^{-8} P+0.894313 \times 10^{-10} P^{2}
\end{aligned}
$$$$
D=0.917151+38.7911 \times 10^{-6} P-1.13552 \times 10^{-8} P^{2}
$$$$
E=100.0
$$

$\mathrm{P}$ is the pressure in $\mathrm{MN} / \mathrm{m}^{2}$
A special software is designed to handle Eqs.(1-7) to recast $n^{2}(\lambda, P)$ under the following form to account for the thermal effects, recalling again Eq.(1) as,

$$
n^{2}(\lambda, T, P)=n^{2}(\lambda, T) f(P, \lambda)
$$

where $f(P, \lambda)$ is found to possess the form:

$f(P, \lambda)=1+R(P, \lambda)$

where:

$$
R(P, \lambda)=\alpha+\beta \lambda+\gamma \lambda^{2}
$$

and:

$$
\left[\begin{array}{c}
\alpha \\
\beta \\
\gamma
\end{array}\right]=\left[\begin{array}{ccc}
1.424 \times 10^{-3} & 1.293 \times 10^{-5} & -5.252 \times 10^{-9} \\
2.477 \times 10^{-6} & -7.419 \times 10^{-7} & 3.442 \times 10^{-9} \\
6.990 \times 10^{-6} & 1.965 \times 10^{-7} & -1.041 \times 10^{-9}
\end{array}\right]\left[\begin{array}{c}
1 \\
P \\
P^{2}
\end{array}\right]
$$

with rms errors of $\alpha, \beta$, and $\gamma$ are $10^{-11}, 10^{-16}$, and $10^{-21}$ respectively over the following ranges:

$$
\begin{aligned}
& 1.3 \leq \lambda, \mu m \leq 1.6 \\
& 0.0 \leq P, M N / m^{2} \leq 300
\end{aligned}
$$

The thermal-dependent refractive index $n(\lambda, T)$ is cast on the same spirit of $[13,14]$.

$$
n^{2}(\lambda, T)=\sum_{i=1}^{3} \frac{A_{i}(T) \lambda^{2}}{\lambda^{2}-\lambda_{i}^{2}(T)}
$$

Based on the data reported by Knauss [15], both the pressure, $\mathrm{P}$, and the temperature, $\mathrm{T}$, are correlated to the depth, h, as:

$$
P=9.918 h-0.2374 \times 10^{-1} h^{2}+0.1922 \times 10^{-3} h^{3}, \mathrm{MN} / \mathrm{m}^{2}
$$

$h \leq 10 \mathrm{~km}$ and

$$
\begin{aligned}
& T=27.2-0.62671 \times 10^{2} h+0.53838 \times 10^{2} h^{2} \\
& -0.20674 \times 10^{2} h^{3}+3.5994 h^{4}-0.23156 h^{5}, \quad{ }^{o} C
\end{aligned}
$$

$h \leq 5.5 \mathrm{~km}$ and $\mathrm{T}=$ constant, $\mathrm{h}>5.5 \mathrm{~km}$.

Equation (1) is the cornerstone in the computation of the dispersion effects and consequently the system capacity; recall that:

$$
\mathrm{n}(\lambda, \mathrm{T}, \mathrm{P})=\mathrm{n}(\lambda, \mathrm{T}) \sqrt{\mathrm{f}(\lambda, \mathrm{P})}=n(\lambda, T) F(\lambda, P)
$$

where

$$
\underset{(6)}{F}(\lambda, P)=1.0+0.5 * R(\lambda, P) \text {. }
$$

Based on the models of Refs. [16,17], the total chromatic dispersion of a single mode fiber, $\tau$, depends on $\mathrm{n}(\lambda, \mathrm{T}, \mathrm{P})$ and its first and second derivatives $\mathrm{n}{ }^{`}$ and $n$ ” w.r.t. $\lambda$, respectively, are given by 
$\mathrm{n}^{`}(\lambda, \mathrm{T}, \mathrm{P})=\mathrm{n}(\lambda, T) F^{`}(\lambda, P)+n^{`}(\lambda, T) F(\lambda, P)$

$\mathrm{n}^{`}(\lambda, \mathrm{T}, \mathrm{P})=\mathrm{n}(\lambda, T) F^{`}(\lambda, P)+n^{\prime}(\lambda, T) F(\lambda, P)+2 n^{`}(\lambda, T) F^{`}(\lambda, P)$

The thermal-dependent spectral losses, $\sigma$, are processed based on the models of Refs. [18-20].

The multi-limitations bit rate (dispersion, losses, and depth) is calculated based on the same spirit of the model of CSELET ${ }^{[20]}$, where it is simplified as:

$$
B_{r}=F_{t} \sqrt{8 \log \frac{B_{u}}{B_{r}} e^{-\left(\sigma L_{f}+\sigma_{m}\right)}}
$$

where

$\begin{array}{ll}\mathrm{F}_{\mathrm{t}} & : \text { Fiber bandwidth }(\mathrm{BW})=0.44 /\left(\tau \mathrm{L}_{\mathrm{f}}\right) \\ \mathrm{B}_{\mathrm{u}} & : \text { Ultimate bit-rate (limitations-free) } \\ \mathrm{L}_{\mathrm{f}} & : \text { Repeater spacing } \\ \sigma_{\mathrm{m}} & : \text { System marginal loss }\end{array}$

In the following section, $\mathrm{B}_{\mathrm{r}}$, will be parametrically processed with special emphasis on the depth of the ocean.

\section{RESULTS AND DISCUSSION}

Taking into account the variations of the under surface ocean pressure and temperature due to the ocean depth, a special software is cast to handle the relevant calculations, namely:

The ocean depth-pressure variations

The ocean depth-temperature variations

The spectral losses and the dispersion effects

The transmitted bit-rate taking into account the above two limitations.

Germania-doped fibers are employed with doping percentage $(x)$ to shift the free-dispersion zone toward the zone of minimum optical losses. The reality of the processed calculations is considered via a marginal loss of $0.25 \mathrm{~dB}$ per repeater spacing, $\mathrm{L}_{\mathrm{f}}$ which is considered in the present case $60 \mathrm{~km}$ due to the amplifier gain. Two cases of interest are processed:

\section{Case 1: Without dispersion cancellation:}

The variation of the set of variables $\left\{\right.$ Bit-rate $\left(B_{r}\right)$, the dispersion $(\tau)$, the fiber bandwidth $(\mathrm{BW})$, the spectral losses $(\sigma)\}$ against the variations of the optical wavelength at different ocean depths are displayed in Figs.1-8 for two types of fibers.

i) pure silica $x=0.0$, and ii) germania doped fiber of $\mathrm{x}=0.2$, with the following set of parameters:

$\Delta \lambda \quad:$ Spectral width $=2.5 \mathrm{~nm}$

$\Delta \mathrm{n} \quad:$ Refractive index difference $=0.0025$

$\mathrm{L}_{\mathrm{f}} \quad:$ Repeater spacing $=60 \mathrm{~km}$

$\sigma_{\mathrm{m}} \quad:$ System marginal loss $=0.25 \mathrm{~dB}$

$\mathrm{B}_{\mathrm{u}}$ :Maximum available bit-rate without any limitations=6.3 Tbit/sec.

These figures clarify that:

1) The spectral losses, Fig.4, decrease with the ocean depth due to the decrease of the ocean temperature.

2) The dispersion-free zone moves towards the third communication window $(\lambda \cong 1.55 \mu \mathrm{m})$, Fig.3. The fiber bandwidth possesses the features as in Fig.2.
3) Consequently according to "a" and "b", the transmitted bit rate possesses the features of Fig. 1 with movable maxima towards the third communication window $(\lambda=1.55 \mu \mathrm{m})$

4) The same features are found at $x=0.2$ but at higher optical wavelengths.

The maxima of the bit rates, $\mathrm{B}_{\text {rmax. }}$ (dispersion-free) are displayed as functions of the ocean depth as well as the corresponding dispersion-free optical wavelength in Figs. 9 and 10 where some sort of saturation (flatness) is shown. Based on Eq.(17), the dispersion-free bit rate $\mathrm{B}_{\text {rmax. }}(\tau=0.0$ and consequently $\mathrm{BW}=\infty)$ is given by:

$B_{r \max .}=B_{u} e^{-\left(\sigma L_{f}+\sigma_{m}\right)}$

which is a losses-limited one.

The variations of the set of controlled parameters $\left[\mathrm{B}_{\mathrm{r}}, \mathrm{WB}, \tau, \sigma\right]$ against the variations of the ocean depth at different optical wavelengths are displayed in Figs.11-20. The main features of these figures are:

1) Flattened ranges of variations of $T, \tau$, and $\sigma$ and consequently, in general, flattened variations of $\mathrm{B}_{\mathrm{r}}$.

2) Due to ripples of the variations, $B_{r}$ possesses more than one maximum value.

3) Whatever the operating optical wavelength, $B_{r}$ increases with the depth due to the decrease of $\mathrm{T}, \sigma, \tau$, and BW. The complicated variations of this case are due to the opposite effects of variations of both $\sigma$ and $\tau$ as in Figs. 21-24.

\section{Case 2: With dispersion cancellation}

In this case, two segments of fibers of numerically equal dispersion bit of opposite sign are spliced in a periodical manner along the cable. Each segment is of 2-km length, thus the dispersion is cancelled each $4 \mathrm{~km}$ along the path.

In such case and from the point of view of dispersion, $\mathrm{L}_{\mathrm{f}}$ is considered $4 \mathrm{~km}$. The same sort of variations are displayed in Figs.25-32, but with the following main features:

1) The dispersion-free wavelength moves towards the third communication window $(\lambda=1.55 \mu \mathrm{m})$

2) The increase of the transmitted bit rates in general is found without sharpening as in Fig.1

3) If compared with the first case, the two cases possess the same maxima as in Figs. 9 and 10.

4) The increase of $x$ shifts the dispersion-free wavelength to higher values, and consequently the bit rate.

\section{CONCLUSION}

The ocean depth is of a special impact in submarine optical fiber cables due to its effects on pressure and temperature and consequently on the physical parameters of the optical fibers such as the optical losses and the dispersion effects. It is found that as the depth increases, the dispersion-free optical wavelength moves toward the third optical communication window, with a maximum value of the transmitted bit rate. Over the range of depth from $1 \mathrm{~km}$ up to $5.5 \mathrm{~km}$, 
the maxima of the transmitted bit rates undergo an approximately flattened shape, where the maxima range is from $10 \mathrm{Gbit} / \mathrm{sec}$, at $\mathrm{x}=0.2$ up to $45 \mathrm{Gbit} / \mathrm{sec}$ at $\mathrm{x}=0.0$ (pure silica). In the case of dispersion cancellation, clarified improvement of the transmitted bit-rate is found. 
Fig. 1 Variations of $B_{r}$ against variations of $\lambda$ at the assumed set of parameters.

Fig. 2 Variations of BW against variations of $\lambda$ at the assumed set of parameters.
Fig. 3 Variations of $\tau$ against variations of $\lambda$ at the assumed set of parameters.

Fig. 4 Variations of $\sigma$ against variations of $\lambda$ at the assumed set of parameters. 
Fig. 5 Variations of $B_{r}$ against variations of $\lambda$ at the assumed set of parameters.

Fig. 6 Variations of BW against variations of $\lambda$ at the assumed set of parameters.
Fig. 7 Variations of $\tau$ against variations of $\lambda$ at the assumed set of parameters.

Fig. 8 Variations of $\sigma$ against variations of $\lambda$ at the assumed set of parameters. 
Fig. 9 Variations of $\lambda_{0}$ against variations of $D$ at the assumed set of parameters.

Fig. 10 Variations of $B_{\text {rmax. }}$ against variations of $D$ at the assumed set of parameters.
Fig. 11 Variations of $B_{r}$ against variations of $D$ at the assumed set of parameters.

Fig. 12 Variations of BW against variations of D at the assumed set of parameters. 
Fig. 13 Variations of $\tau$ against variations of $D$ at the assumed set of parameters.

Fig. 14 Variations of $\sigma$ against variations of $D$ at the assumed set of parameters.
Fig. 15 Variations of $T$ against variations of $D$ at the assumed set of parameters. 
Fig. 17 Variations of $B_{r}$ against variations of $D$ at the assumed set of parameters.

Fig. 18 Variations of BW against variations of D at the assumed set of parameters.
Fig. 19 Variations of $\tau$ against variations of $D$ at the assumed set of parameters.

Fig. 20 Variations of $\sigma$ against variations of $D$ at the assumed set of parameters. 
Fig. 21 Variations of $\tau$ against variations of $\mathrm{T}$ at the assumed set of parameters.

Fig. 22 Variations of $\sigma$ against variations of at the assumed set of parameters.
Fig. 23 Variations of $\tau$ against variations of $\mathrm{T}$ at the assumed set of parameters.

Fig. 24 Variations of $\sigma$ against variations of $T$ at the assumed set of parameters. 
Fig. 25 Variations of $B_{r}$ against variations of $\lambda$ at the assumed set of parameters.

Fig. 26 Variations of BW against variations of $\lambda$ at the assumed set of parameters.
Fig. 27 Variations of $B_{r}$ against variations of $\lambda$ at the assumed set of parameters.

Fig. 28 Variations of BW against variations of $\lambda$ at the assumed set of parameters. 
Fig. 29 Variations of $B_{r}$ against variations of $D$ at the assumed set of parameters.

Fig. 30 Variations of BW against variations of D at the assumed set of parameters.
Fig. 31 Variations of $B_{r}$ against variations of $D$ at the assumed set of parameters.

Fig. 32 Variations of BW against variations of D at the assumed set of parameters. 


\section{REFERENCES}

[1] P. K. Runge, "Undersea Lightwave Systems", Optics \& Photonics News, Vol.1, No.11, pp.9-12, Nov. 1991.

[2] Y. Negishi, Y. Miyajima, O. Kawata, and N. Yoshizawa, "First Sea Trial of $1.5 \mu \mathrm{m}$ Submarine Optical Fiber Cable", J. Lightwave Technol., Vol.6, No.2, pp. 281-286, February 1988.

[3] S. Yamamoto, H. Sakaguchi, M. Nunokawa, and Y. Iwamoto, "1.55 $\mu \mathrm{m}$ Fiber -Optic Transmission Experiments for Long-Span Submarine Cable System Design", J. Lightwave Technol., Vol.6, No.3, pp. 380391, March 1988.

[4] Y. Namihira, Y. Horiuchi, S. Ryu, K. Mochiruki, and H. Wakabayash, "Dynamic Polarization Fluctuations Characteristics of Optical Fiber Submarine Cable Under Various Environmental Conditions", J. Lightwave Technol., Vol.6, No.5, pp. 728-738, May 1988.

[5] J. Thiennot, F. Pirio, and J. B. Thomine, "Optical Undersea Cable System Trends", Proc. IEEE, Vol. 81, No. 11, pp.1610-1622, Nov.1993.

[6] H. Taga, N. Edagawa, S. Yamamoto, and S. Akiba, "Recent Progress in Amplified Undersea Systems", J. Lightwave Technol., Vol.13, No.5, pp. 829-840, May 1995.

[7] N. S. Bergano, "Undersea Lightwave Transmission Systems Using Er-Doped Fiber Amplifiers", Optics \& Photonics News, pp. 9-14, January 1993.

[8] M. Sumida, S. Furukawa, K. Tanaka, and M. Aiki, "High-Accurate Fault Location Technology Using FSK-ASK Probe Backscattering Reflectometry in Optical Amplifier Submarine Transmission Systems", J. Lightwave Technol., Vol.14, No.10, pp. 2108-2116, October, 1996.

[9] M. Murakami, T, Takashi, M. Aoyama, T. Imai, M. Amemija, M. Sumida, and M. Aiki, "System Performance Evaluation of the FSA Submarine Optical Amplifier System", J. Lightwave Technol., Vol.14, No.12, pp. 2657-2671, December 1996.

[10] J. Adame and P. Estenes, "ISO 9000 and Undersea Fiber Cable: Meeting the Standard Makes a Difference, "Photonics Spectra", pp. 117-122, September, 1992.

[11] M. Murakami, T. Imai, and M. Aoyama, "A Remote Supervisory System Based on Subcarrier Overmodulation for Submarine Optical Amplifier Systems", J. Lightwave Technol., Vol.14, No.5, pp. 617-677, May 1996.

[12] G. Ghosh, and H. Yajima, "Pressure-Dependent Sellmeier Coefficients and Material Dispersions for Silica Fiber Glass", J. Lightwave Technol., Vol.16, No.11, pp. 2002-2005, November 1998.

[13] J. W. Fleming , "Dispersion in $\mathrm{GeO}_{2}-\mathrm{SiO}_{2}$ Glasses", Appl. Opt., Vol. 23, No. 24, pp. 4488-4493, 15 Dec., 1984.

[14] A. A. Aoul-Enein, "Termal-Dependent Chromatic Dispersion in Lightwave System", Proc. 14 the Intern. Conf. Statistics, Computer Science, Social and Demographic Research, Cairo Egypt, Vol.2, pp.195211, March 1989.

[15] J. A. Knauss, Introduction to Physical Oceanography, 2/e, Prentice Hall, Inc., U.S.A 1997.

[16] L. B. Jeunhomme, Single-Mode Optical Fiber, Marcel Dekker, Inc., N.Y., USA, 1983.
[17] M. J. Adams, An Introduction to Optical Waveguides; John Wiley \& Sons, N.Y, UsA 1981, Ch.7.

[18] S. S. Walker, "Rapid Modelling and Estimation of Total Spectral Loss in Optical Fibers", J. Lightwave Technol., Vol. 4, No.8, pp.1125-1137, Aug. 1996.

[19] A. A. Aboul Enein, F. Z. El-Halafawy, M. H. A. Hassan, A. A. Mohammed, "Thermal Environmental Effects", Alex. Eng. J., Alex. Univ., Vol.28, pp.169$183,1989$.

[20] CSELET, Turino, ITALY, Optical Fiber Communications, 1/e, McGraw-Hill Inc., USA, 1981.

\section{BIOGRAPHY}

Dr. A. A. Mohammed was born in Sohag, Egypt, 1959. He has obtained his B.Sc., M.Sc., and Ph.D. in Electronic Engineering, from Faculty of Electronic Engineering, Menouf, Menoufia University, Egypt, in 1986, 1991 and 1994, respectively. His interest is in the area of optical communications: optical sources, optical amplifiers, optical cables, optical networks, different propagation phenomena in different optical channels, etc. Other area of interest is the computer-software using different languages and different techniques. 